\title{
Metronidazole-induced encephalopathy in a patient with Crohn's disease
}

\author{
Jihye Kim ${ }^{1}$, Jaeyoung Chun ${ }^{1}$, Jae Yong Park ${ }^{1}$, Seung Wook Hong ${ }^{1}$, Joo Young Lee ${ }^{1}$, Jin Woo Kang ${ }^{1}$, \\ Seongjun Hwang ${ }^{2}$, Sang-Bae Ko ${ }^{3}$, Jong Pil Im ${ }^{1}$, Joo Sung Kim ${ }^{1}$ \\ ${ }^{1}$ Department of Internal Medicine and Liver Research Institute, Seoul National University College of Medicine, Seoul, \\ Departments of ${ }^{2}$ Internal Medicine and ${ }^{3}$ Neurology, Seoul National University College of Medicine, Seoul, Korea
}

Metronidazole is a widely used antibiotic for the treatment of anaerobic bacterial infections. Metronidazole-induced encephalopathy (MIEP) is a rare but potentially reversible disease. The mechanism of MIEP remains unclear, and differences in the neurotoxic effects of oral versus intravenous (IV) metronidazole administration have not yet been determined. We report the case of a Crohn's disease (CD) patient who experienced encephalopathy immediately after a single IV dose of metronidazole following long-term exposure to the oral form of the drug. The 64-year-old man with intractable CD experienced a sudden change in mental status, aphasia, and muscle weakness after IV administration of metronidazole. He had previously taken metronidazole orally for 13 years and received intermittent IV metronidazole treatments for CD exacerbation. Brain magnetic resonance imaging (MRI) showed high-intensity signals in the bilateral medial thalamus and the midbrain and pontine tegmentum on fluid-attenuated inversion recovery images. After discontinuation of metronidazole, the high-intensity brain MRI signals resolved and the patient's mental status dramatically improved; however, the patient exhibited mild cognitive dysfunction 2 months after the onset of encephalopathy. (Intest Res 2017;15:124-129)

Key Words: Metronidazole; Drug side effects; Metabolic encephalopathy; Crohn disease

\section{INTRODUCTION}

Crohn's disease (CD) is an immunological disorder characterized by a relapsing-remitting clinical course. ${ }^{1}$ Antibiotics are effective in the treatment of $\mathrm{CD}$, especially in patients with complicated fistulas or abscesses. ${ }^{2}$ Increasing evidence suggests that dysregulated gut microbiota play a role in the pathogenesis of $\mathrm{CD}^{3,4}$ Metronidazole is a widely used antibiotic in patients with CD. ${ }^{5}$ However, metronidazole may elicit neurotoxic effects including peripheral neuropathy, ataxia, seizure, and encephalopathy. Metronidazole-induced en-

Received May 10, 2015. Revised July 2, 2015

Accepted July 22, 2015.

Correspondence to Jaeyoung Chun, Department of Internal Medicine and Liver Research Institute, Seoul National University College of Medicine, 101 Daehak-ro, Jongno-gu, Seoul 03080, Korea. Tel: +82-2-740-8112, Fax: +822-743-6701,E-mail:j40479@gmail.com

Financial support: None. Conflict of interest: None. cephalopathy (MIEP) is a very rare but potentially reversible disease. ${ }^{6,7}$ The exact mechanism of MIEP remains unclear. Here, we report the case of a CD patient who experienced encephalopathy immediately after a single intravenous (IV) dose of metronidazole following long-term exposure to the oral form of the drug.

\section{CASE REPORT}

A 64-year-old man who had been diagnosed with CD 17 years previously presented with altered mental status. He received sulfasalazine and azathioprine as maintenance treatment for CD and was intermittently treated with prednisolone to achieve remission after flare-ups. He had also received infliximab for the previous 3 years because of refractoriness to conventional treatment. Fourteen years before beginning infliximab treatment, the patient underwent ileocecectomy due to intractable active CD. Nine years after

\footnotetext{
๑ Copyright 2017. Korean Association for the Study of Intestinal Diseases. All rights reserved.

This is an Open Access article distributed under the terms of the Creative Commons Attribution Non-Commercial License (http://creativecommons.org/licenses/by-nc/4.0)

which permits unrestricted non-commercial use, distribution, and reproduction in any medium, provided the original work is properly cited.
} 
his first surgical treatment for active $\mathrm{CD}$, he underwent right hemicolectomy and small bowel segmental resection to treat an intra-abdominal abscess communicating with multiple fistulas. He underwent three additional bowel resections because of a recurrent wound infection with enterocutaneous fistulas. The length of his remaining small intestine was $120 \mathrm{~cm}$.

The patient had taken $250 \mathrm{mg}$ of metronidazole orally three times daily beginning 13 years previously. After 8 years of treatment, however, he stopped taking metronidazole orally because of the side effect of peripheral neuropathy. He received IV metronidazole treatments three times over the following 5 years when he experienced complications such as enterocutaneous fistulas or abscesses. Six months before the last admission, however, he restarted and continued oral metronidazole treatment for active CD. The cumulative dose

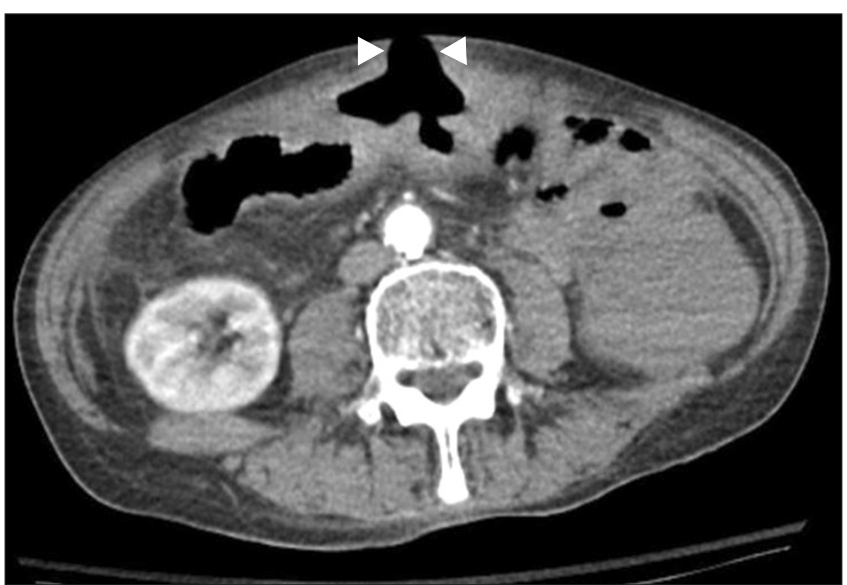

Fig. 1. Abdominal CT finding. CT shows a large enterocutaneous fistula in the middle portion of the abdomen (arrowheads).
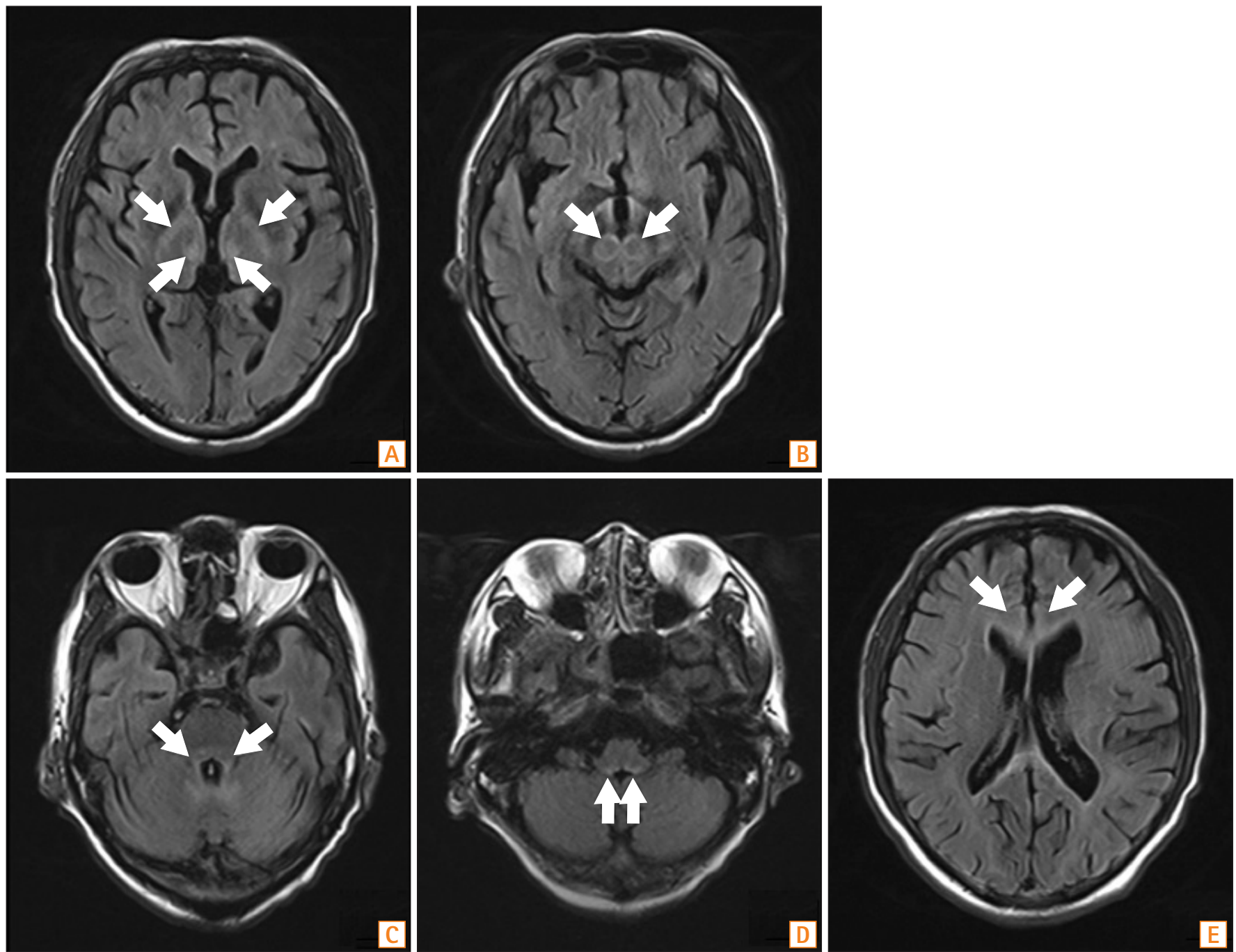

Fig. 2. Brain MRI findings. Fluid-attenuated inversion recovery MRI shows high-intensity lesions in the bilateral medial thalamus (A), midbrain tegmentum (B), pontine tegmentum (C), fourth ventricle and dentate nucleus (D), and subcortical white matter (E) (arrows). 
of metronidazole over the 6 months prior to the last admission was $111.75 \mathrm{~g}$. His lifetime cumulative dose of metronidazole was approximately 2,000 g.

The patient visited the hospital emergency department complaining of an abdominal wound problem which had been aggravated 2 days previously. He presented with pain, redness, and swelling surrounding the wound. Upon examination, he exhibited mild tenderness in the middle to lower abdomen. Initial laboratory findings showed elevated CRP $(4.3 \mathrm{mg} / \mathrm{dL})$, total bilirubin $(4.9 \mathrm{mg} / \mathrm{dL})$, and direct bilirubin $(3.7 \mathrm{mg} / \mathrm{dL}$ ), as well as prolonged PT (INR of 2.07). Other laboratory findings were as follows: white blood cell count $8,100 / \mathrm{mm}^{3}$, hemoglobin level $12.4 \mathrm{~g} / \mathrm{dL}$, platelet count $140 \times 10^{9} / \mathrm{L}$, cholesterol level $62 \mathrm{mg} / \mathrm{dL}$, total protein level 5.6 $\mathrm{g} / \mathrm{dL}$, albumin level $2.8 \mathrm{~g} / \mathrm{dL}$, BUN level $10 \mathrm{mg} / \mathrm{dL}$, serum creatinine level $0.57 \mathrm{mg} / \mathrm{dL}$, serum AST level $22 \mathrm{IU} / \mathrm{L}$, serum ALT level 16 IU/L, and serum ALP level $61 \mathrm{IU} / \mathrm{L}$. Test results for all serological markers of hepatitis B and $\mathrm{C}$ were negative. Abdominal CT scans revealed a large enterocutaneous fistula in the middle portion of the patient's abdomen (Fig. 1).

Considering the shortness of his small bowel, the patient received conservative treatment including parenteral nutrition and antibiotics for the treatment of the wound infection and enterocutaneous fistula. Metronidazole was administered intravenously during fasting.

Prior to IV metronidazole administration, the patient could follow commands and showed no motor weakness (Glasgow coma scale score of 15). However, he abruptly
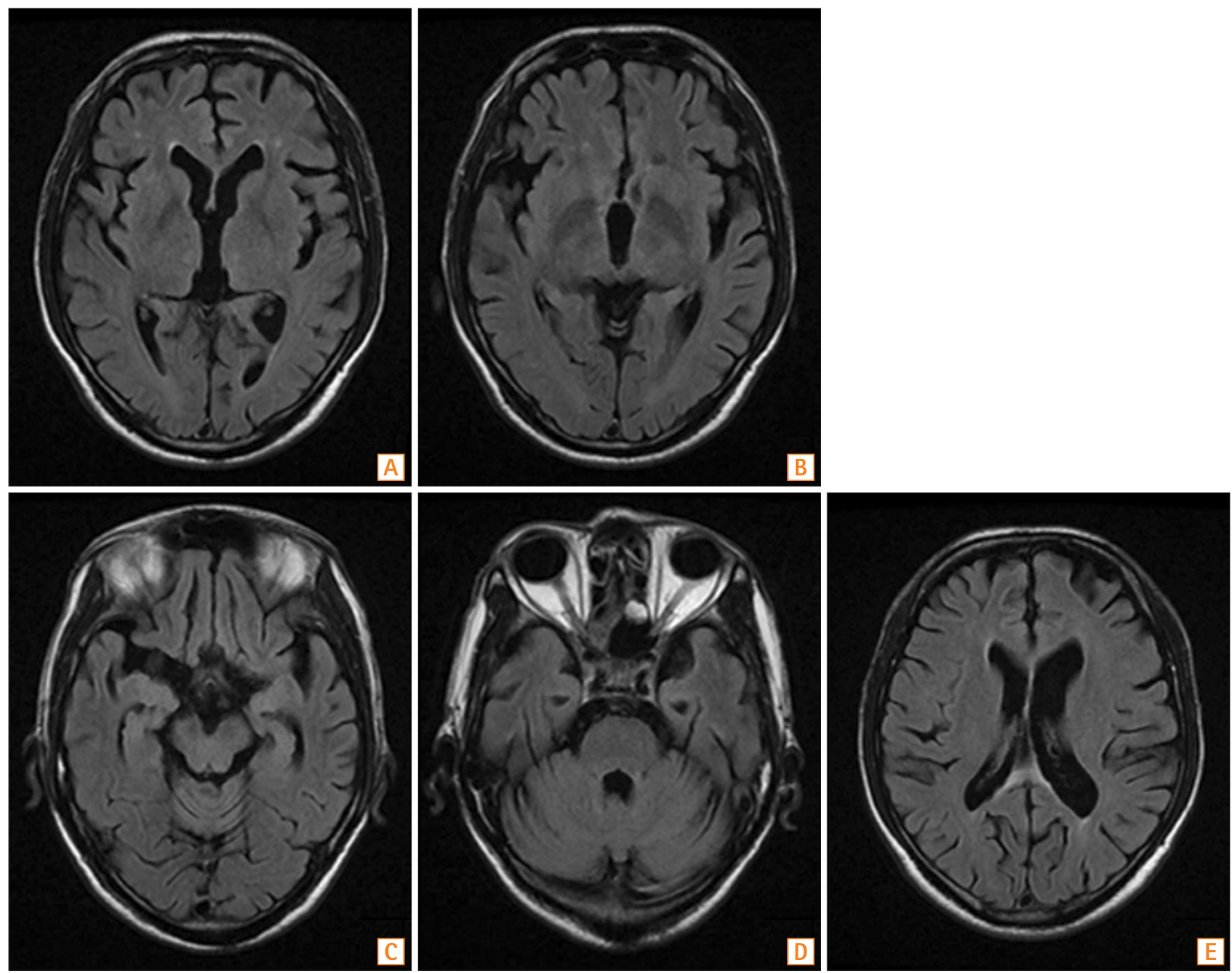

Fig. 3. Follow-up brain MRI findings. MR images obtained 7 days after metronidazole discontinuation. A fluid-attenuated inversion recovery image shows the improvement of lesions in the bilateral medial thalamus $(A, B)$; midbrain tegmentum $(C)$; pontine tegmentum, fourth ventricle, and dentate nucleus (D); and subcortical white matter (E). 
became comatose (Glasgow coma scale score of 8 ) 2 hours after receiving a single $500 \mathrm{mg}$ IV dose of metronidazole. On neurological examination, the patient could not open his eyes upon painful stimulation but could localize the pain bilaterally. All brainstem reflexes were normal. However, bilateral Babinski signs were observed, suggestive of bilateral diffuse cortico-subcortical lesions. The pupillary light reflex was intact, and there was no limitation of extraocular muscle movement. The patient's vital signs were normal at the time of metronidazole administration, with a blood pressure of 139/82 $\mathrm{mmHg}$, heart rate of 86 beats per minute, respiratory rate of 18 breaths per minute, and body temperature of $36.6^{\circ} \mathrm{C}$. Two hours after IV metronidazole administration, however, his blood pressure was $160 / 96 \mathrm{mmHg}$ and his heart rate was 133 beats per minute.

The rapid adrenocorticotropic hormone stimulation test revealed a normal response. The patient's brain CT scan was unremarkable. However, fluid-attenuated inversion recovery MRI showed multiple high-intensity lesions in the bilateral subcortical white matter, medial thalamus, midbrain and pontine tegmentum, bilateral middle cerebellar peduncles, and cerebellar dentate nucleus (Fig. 2). Based on clinical and radiological findings, a tentative diagnosis of MIEP was made. Metronidazole treatment was discontinued and replaced with IV piperacillin/tazobactam. A megadose of IV thiamine (500 $\mathrm{mg}$ ) was administered three times daily for 8 days. The patient was subsequently maintained on $50 \mathrm{mg}$ of thiamine daily.

Seven days after discontinuing metronidazole treatment, the patient was able to track moving objects. On day 8 , a follow-up brain MRI showed more clearly visible diffuse

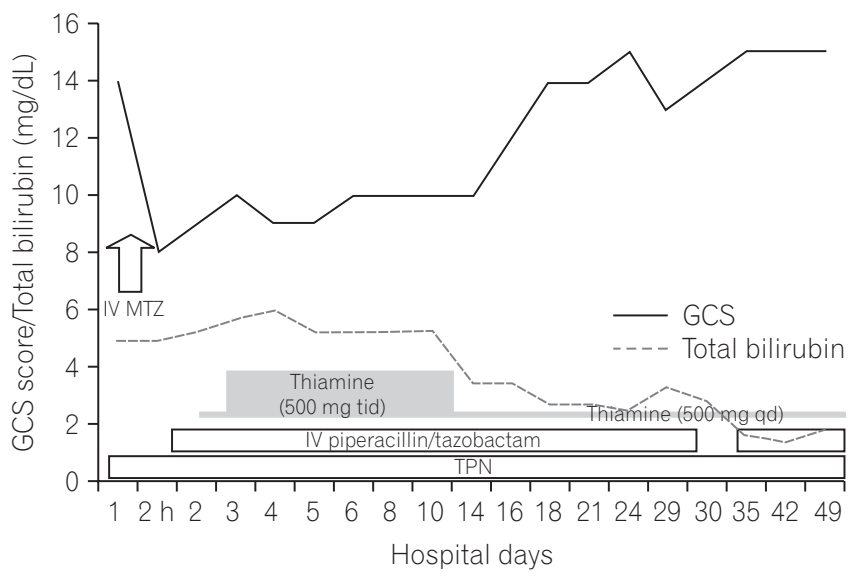

Fig. 4. Serial results of the Glasgow coma scale (GCS) and total bilirubin testing after cessation of metronidazole (MTZ) treatment. IV, intravenous; tid, three times a day; qd, every day; TPN, total parenteral nutrition. bilateral subcortical white matter lesions. However, the extent of the previously noted hyperintense lesions was decreased in the thalamus, brainstem, and cerebellar dentate nucleus (Fig. 3). On day 12, the patient showed a significant improvement in his level of consciousness and was able to follow verbal commands and answer simple questions. Two months after the onset of MIEP, he was able to talk and walk unassisted. However, he still showed mild cognitive dysfunction. He received a score of 15 on the Korean version of the mini-mental state examination (normal range, 25-30) and demonstrated severe frontal dysfunction, as indicated by a score of 7 on the Frontal Assessment Battery (range, 0-18) (Fig. 4).

\section{DISCUSSION}

Metronidazole is associated with declines in CDAI scores and a reduced CD recurrence rate after surgery. ${ }^{8}$ It is well known that metronidazole is comparatively safe at the usual dosages. However, various neurological adverse events, including peripheral neuropathy, cerebellar dysfunction, and seizure, can develop. MIEP is one of the most serious adverse events associated with metronidazole treatment; however, it has a favorable prognosis after drug cessation. The long-term safety of large cumulative doses of metronidazole has not been elucidated. In previous reports, patients who experienced MIEP or cerebellar ataxia were not exposed to larger cumulative doses of metronidazole than that received by our patient; however, the association between neurotoxicity and the cumulative dose or duration of metronidazole treatment remains controversial. ${ }^{9,10}$ Metronidazole-induced neurotoxicity typically occurs at doses exceeding $2 \mathrm{~g} /$ day for prolonged periods. ${ }^{7,9}$ Thus, our patient may have been at high risk of MIEP because of an extremely high cumulative dose of metronidazole received over a period of 13 years. Previous history of long-term exposure to metronidazole should be considered to prevent neurotoxic effects such as MIEP in patients who require repeated treatment.

Previous studies have shown that brain lesions identified by MRI in MIEP patients are typically located in the cerebellar dentate nucleus, dorsal medulla, dorsal pons, midbrain, and splenium of the corpus callosum, with bilateral and symmetrical involvement. ${ }^{11}$ Thus, the brain MRI findings in this case were consistent with changes associated with MIEP.

In this case, MIEP developed immediately after IV administration of a single dose of metronidazole following long-term oral metronidazole treatment. Differences in the neurotoxic effects of oral versus IV metronidazole adminis- 
tration are not yet understood. A previous study showed that the upper part of the small intestine is the main site of metronidazole absorption. ${ }^{12}$ Manafi et al. ${ }^{13}$ found that absorption of orally administered metronidazole was significantly reduced by postoperative ileus. Therefore, a short bowel may be related to poor absorption of both nutrients and the metronidazole. The peak serum level of metronidazole after IV administration in patients with a short bowel may be higher than that after oral administration.

Because metronidazole is mainly metabolized in the liver, severe liver dysfunction increases the drug's half-life and increases the risk of MIEP. ${ }^{14}$ In our case, there were no clinical signs suggesting chronic liver disease or hepatic encephalopathy, although the patient had cholestasis and PT prolongation at admission. In addition, the abdominal CT scan showed neither cirrhotic changes nor mechanical biliary obstruction. Thus, these transient abnormalities revealed by the liver function test may have been related to impaired hepatocyte transport function and systemic infection caused by bowel inflammation and bacterial overgrowth. ${ }^{15}$ Liver function test results were stable for 2 weeks after admission and gradually returned to normal. In contrast, the patient's mental status began to improve within a week after cessation of metronidazole treatment. This temporal relationship supports the diagnosis of MIEP rather than hepatic encephalopathy.

In this case, Wernicke's encephalopathy (WE) may be another possible diagnosis, as acute WE has clinical symptoms and brain MRI findings similar to those of MIEP. Metronidazole metabolites inhibit thiamine pyrophosphokinase, and the resulting thiamine antagonism may also play a role in the pathogenesis of MIEP. ${ }^{16}$ However, the patient displayed altered mental status immediately after IV administration of metronidazole. The temporal relationship between metronidazole administration and onset of neurological symptoms suggests a diagnosis of MIEP rather than WE. Moreover, the patient showed no clinical signs of oculomotor dysfunction such as nystagmus and conjugate gaze palsy, which are typical clinical features of WE. ${ }^{17}$ Brain MRI revealed diffuse high-intensity lesions in the bilateral subcortical white matter, medial thalamus, midbrain and pontine tegmentum, bilateral middle cerebellar peduncles, and cerebellar dentate nucleus, which are compatible with a diagnosis of MIEP. Lesions in the dentate nucleus and red nucleus can also be found in patients with $\mathrm{WE}$, although these findings are atypical. ${ }^{18}$ The most common structures affected by WE, as demonstrated by MRI, are the medial thalamus and periventricular regions of the third ventricle. ${ }^{19,20}$ In a systematic review of 64 patients with MIEP, MRI revealed subcortical white matter lesions in approximately $50 \%$ of patients with altered metal status. ${ }^{9}$ However, high-intensity lesions in the bilateral subcortical white matter have not been detected in patients with WE., ${ }^{9,11,18-20}$ These findings strongly suggest that MIEP is the primary cause of altered mental status in this case. Nonetheless, the possibility of concomitant WE could not be completely excluded because the patient was at high risk of thiamine deficiency due to malnutrition resulting from his short bowel status and was treated with thiamine. Moreover, the subtle cardiovascular signs observed in our patient, such as tachycardia, are frequently also observed in patients with acute WE. Because the patient's serum thiamine level was not measured before thiamine replacement therapy, the possibility of concomitant WE may be a limitation in this case study.

We report the first case of a patient with CD who experienced encephalopathy immediately after IV administration of a single dose of metronidazole following long-term oral metronidazole treatment. MIEP may be a rare complication in CD patients, especially those at risk of malnutrition or who have received a high cumulative dose of metronidazole over a long period. Therefore, evaluation of nutritional status is very important in patients with IBD to prevent irreversible complications such as WE. ${ }^{21,22}$

\section{REFERENCES}

1. Kim DH, Cheon JH, Park JJ, et al. Clinical outcomes and predictive factors for response after the first course of corticosteroid therapy in patients with Crohn's disease. Gut Liver 2013;7:5865.

2. Löfmark S, Edlund C, Nord CE. Metronidazole is still the drug of choice for treatment of anaerobic infections. Clin Infect Dis 2010;50 Suppl 1:S16-S23.

3. Han DS. Current status and prospects of intestinal microbiome studies. Intest Res 2014;12:178-183.

4. Kim JM. Antimicrobial proteins in intestine and inflammatory bowel diseases. Intest Res 2014;12:20-33.

5. Lamp KC, Freeman CD, Klutman NE, Lacy MK. Pharmacokinetics and pharmacodynamics of the nitroimidazole antimicrobials. Clin Pharmacokinet 1999;36:353-373.

6. Groothoff MV, Hofmeijer J, Sikma MA, Meulenbelt J. Irreversible encephalopathy after treatment with high-dose intravenous metronidazole. Clin Ther 2010;32:60-64.

7. Boyce EG, Cookson ET, Bond WS. Persistent metronidazoleinduced peripheral neuropathy. DICP 1990;24:19-21.

8. Prantera C, Zannoni F, Scribano ML, et al. An antibiotic regimen for the treatment of active Crohn's disease: a randomized, controlled clinical trial of metronidazole plus ciprofloxacin. Am J Gastroenterol 1996;91:328-332. 
9. Kuriyama A, Jackson JL, Doi A, Kamiya T. Metronidazoleinduced central nervous system toxicity: a systematic review. Clin Neuropharmacol 2011;34:241-247.

10. Patel K, Green-Hopkins I, Lu S, Tunkel AR. Cerebellar ataxia following prolonged use of metronidazole: case report and literature review. Int J Infect Dis 2008;12:e111-e114.

11. Seok JI, Yi H, Song YM, Lee WY. Metronidazole-induced encephalopathy and inferior olivary hypertrophy: lesion analysis with diffusion-weighted imaging and apparent diffusion coefficient maps. Arch Neurol 2003;60:1796-1800.

12. Idkaidek NM, Najib NM. Enhancement of oral absorption of metronidazole suspension in humans. Eur J Pharm Biopharm 2000;50:213-216.

13. Manafi A, Panjehshahin MR, Saravi MG, Emami SA, Forootan SK. Reduced bioavailability of oral metronidazole in postoperative ileus. Iran Red Crescent Med J 2007;9:129-132.

14. Yamamoto T, Abe K, Anjiki H, Ishii T, Kuyama Y. Metronidazoleinduced neurotoxicity developed in liver cirrhosis. J Clin Med Res 2012;4:295-298.

15. Chand N, Sanyal AJ. Sepsis-induced cholestasis. Hepatology 2007;45:230-241.

16. Zuccoli G, Pipitone N, Santa Cruz D. Metronidazole-induced and Wernicke encephalopathy: two different entities sharing the same metabolic pathway? AJNR Am J Neuroradiol 2008;29:E84.
17. Harper CG, Giles M, Finlay-Jones R. Clinical signs in the Wernicke-Korsakoff complex: a retrospective analysis of 131 cases diagnosed at necropsy. J Neurol Neurosurg Psychiatry 1986;49:341-345.

18. Manzo G, De Gennaro A, Cozzolino A, Serino A, Fenza G, Manto A. MR imaging findings in alcoholic and nonalcoholic acute Wernicke's encephalopathy: a review. Biomed Res Int 2014;2014:503-596.

19. Kim E, Na DG, Kim EY, Kim JH, Son KR, Chang KH. MR imaging of metronidazole-induced encephalopathy: lesion distribution and diffusion-weighted imaging findings. AJNR Am J Neuroradiol 2007;28:1652-1658.

20. Zuccoli G, Pipitone N. Neuroimaging findings in acute Wernicke's encephalopathy: review of the literature. AJR Am J Roentgenol 2009;192:501-508.

21. Shin IS, Seok H, Eun YH, et al. Wernicke's encephalopathy after total parenteral nutrition in patients with Crohn's disease. Intest Res 2016;14:191-196.

22. Yoon SM. Micronutrient deficiencies in inflammatory bowel disease: trivial or crucial? Intest Res 2016;14:109-110. 